Preclinical evaluation of the simultaneous inhibition of MCL-1 and BCL-2 with the combination of S63845 and venetoclax in multiple myeloma

Apoptotic evasion has been postulated as one of the main mechanisms of multiple myeloma (MM) cell survival. ${ }^{1,2}$ The intrinsic apoptotic pathway, tightly regulated by the BCL-2 protein family, is initiated by intracellularly sensed stress signals and ultimately leads to the permeabilization of the outer mitochondrial membrane. Tumor cells can keep this pathway inactivated, in part, through the overexpression of BCL-2, BCL-X $\mathrm{X}_{\mathrm{L}}$ or MCL-1 antiapoptotic proteins, ${ }^{3}$ which bind to and sequester proapoptotic proteins (e.g. BIM, NOXA, PUMA), thereby eluding apoptosis. Venetoclax is a drug that selectively binds to BCL-2, impeding its activity as an inhibitor of pro-apoptotic proteins. ${ }^{4}$ In $\mathrm{MM}$, a phase I clinical trial of venetoclax in monotherapy (clinicaltrials.gov identifier: 01794520) has been effective, predominantly in the subgroup of patients harboring the $\mathrm{t}(11 ; 14)$ translocation.

Although co-dependencies with BCL-2 and BCL-X $\mathrm{L}$ have
A

A MM.1S $[t(14 ; 16) ;+1 q]$

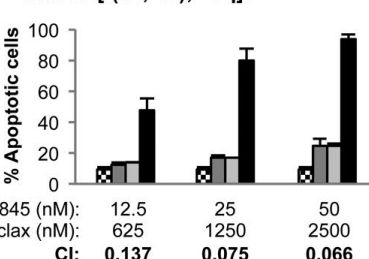

JJN3 [t(14;16); +1q]:

00

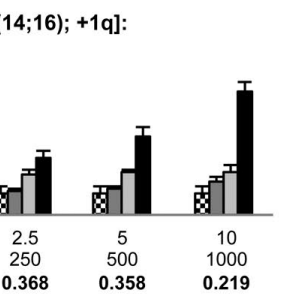

RPMI-8226 [t(14;16); +1q]:

NCl-H929 [t(4;14); +1q]:

$\begin{array}{lll}\mathbf{0 . 3 6 8} & \mathbf{0 . 3 5 8} & \mathbf{0 . 2 1 9}\end{array}$

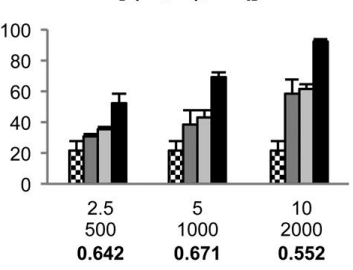

KMS12-BM [t(11;14); +1q]:
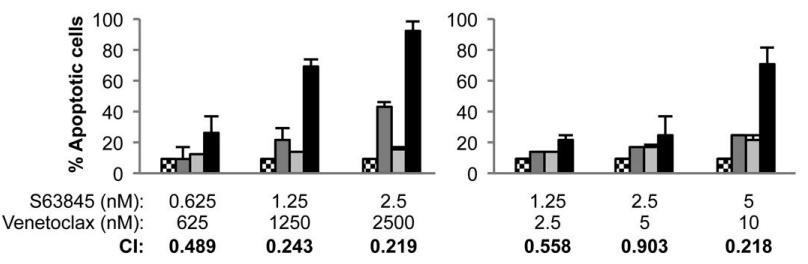

Q Control

$\square \mathrm{S} 63845$

$\square$ Venetoclax

- S63845+Venetoclax

B

\title{
MM.1S:
}

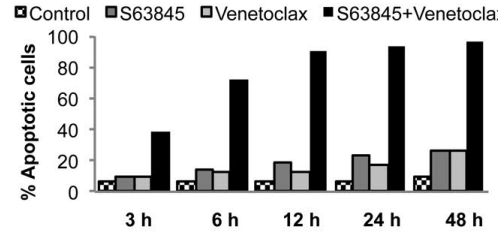

C

Patient 1 [t(11;14)]

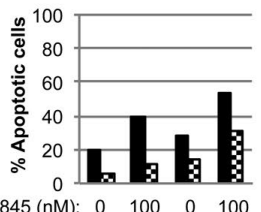

$\mathrm{S} 63845(\mathrm{nM}): 0 \begin{array}{llll}0 & 100 & 0 & 100\end{array}$ $\begin{array}{ccccc}\text { Venetoclax }(\mathrm{nM}): & 0 & 0 & 100 & 100\end{array}$

\section{Pationt 5}

[+1q]

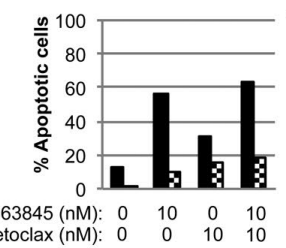

Patient 2
[t(11;14)]

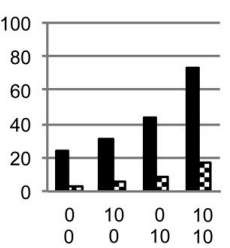

Patient 6

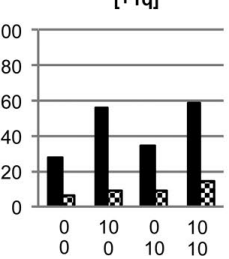

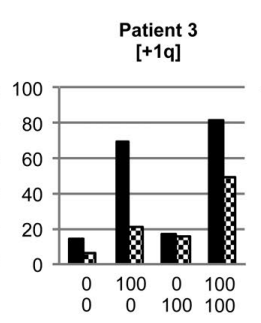

Patient 7

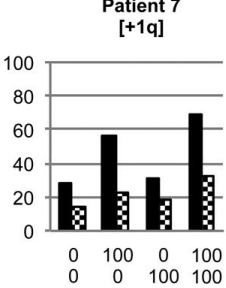

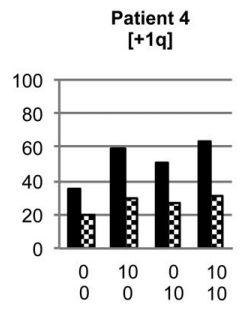

Plasma cells 国 Lymphocytes

Figure 1. S63845 strongly synergizes with venetoclax in vitro. (A) Multiple myeloma (MM) cell lines were exposed to increasing doses of S63845+venetoclax for 48 hours (h), using a constant drug ratio combination design for each cell line. Apoptosis induction was analyzed by flow cytometry after Annexin-V binding and propidium iodide micromolar staining as represented in the graphs, and combination indices (Cl) were calculated with the Calcusyn software (see also Online Supplementary Figure S2). A Cl of 1 indicates an additive effect, $\mathrm{Cl}<1$ a synergistic effect and $\mathrm{Cl}<1$ antagonism. (B) MM.1S cells were treated with $\mathrm{S} 6384550 \mathrm{nM}$ and venetoclax $2.5 \mathrm{nM}$ for 3, 6, 12, 24 and $48 \mathrm{~h}$, and the induction of apoptosis was assessed at indicated time points. (C) Bone marrow cells from eight MM patients were incubated with $\mathrm{S} 63845$ and venetoclax as single agents and in combination at indicated doses for $24 \mathrm{~h}$. Apoptosis induction was

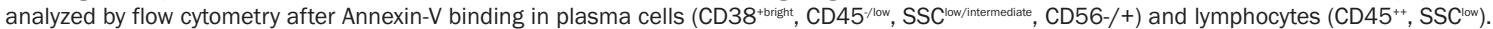


A

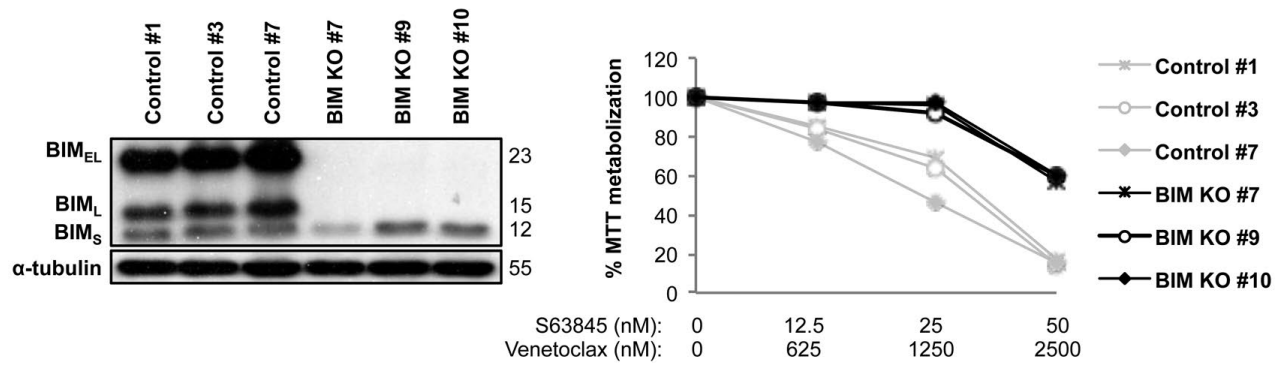

B

MM.1S:
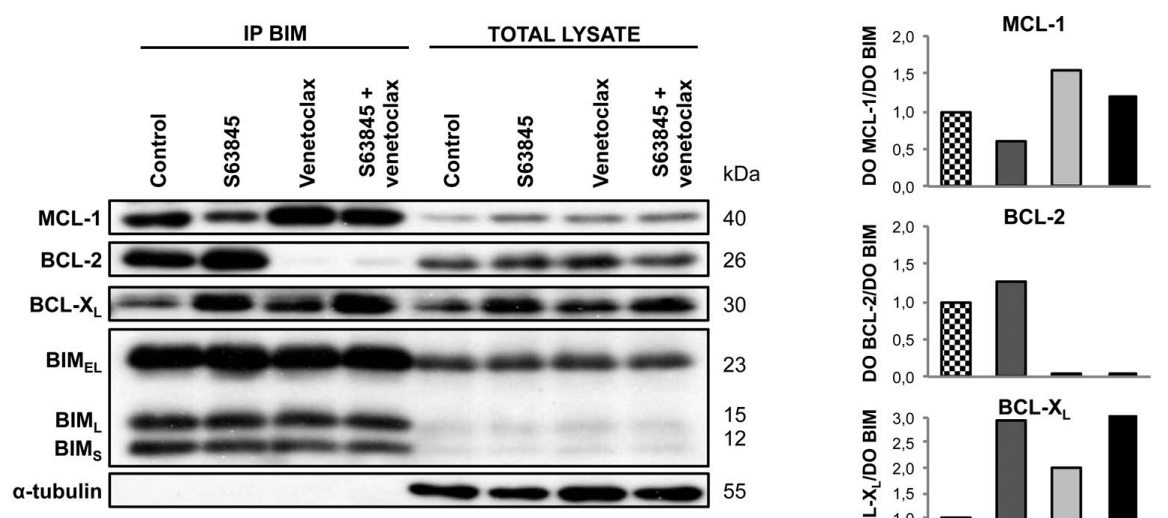

C

KMS12-BM:
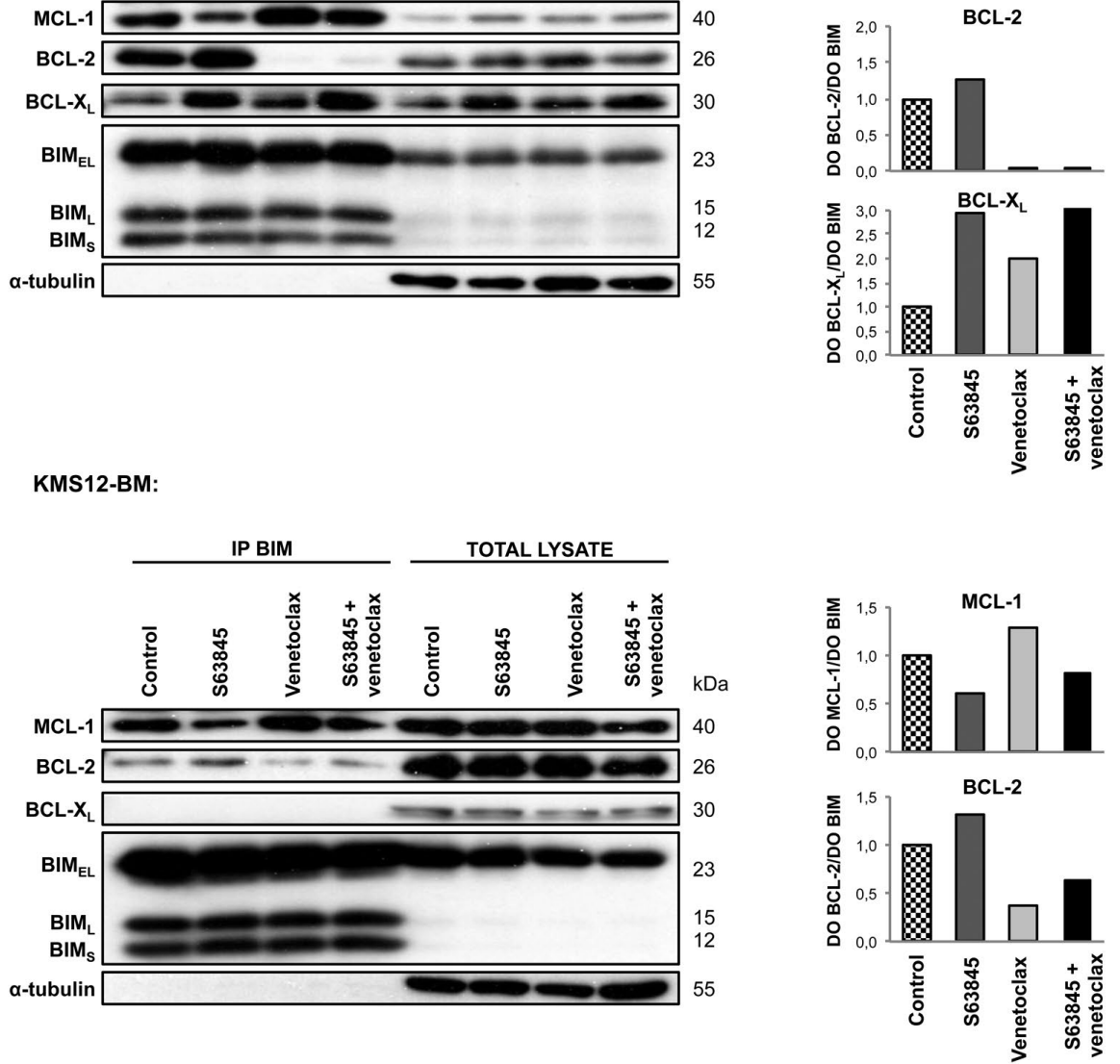

Figure 2. The 563845 + venetoclax combination impairs the interactions of MCL-1 and BCL-2 with the pro-apoptotic protein BIM. (A) BIM shows three major isoforms: BIMєь, BIM ing a guide RNA and a Cas9 enzyme (Integrated DNA Technologies)], using the Neon Transfection System (Thermo Fisher Scientific) and subsequent single cell sorting. Clones KO for BIMEL and BIML isoforms and control clones (electroporated with the Cas 9 enzyme only) were exposed to increasing doses of S63845+venetoclax for 24 hours, keeping a constant 1:50 S63845:venetoclax ratio. Cell viability was analyzed by MTT assay. (B and C) MM.1S and KMS12$\mathrm{BM}$ cell lines (least sensitive and most sensitive to $\mathrm{S63845}$ and venetoclax) were respectively treated with S63845 (12.5 and $2 \mathrm{nM}$ ) and venetoclax (625 and $4 \mathrm{nM}$ ), in monotherapy or in combination for 24 hours (S63845 and venetoclax doses were adjusted for each cell line so that the combination would induce $13-25 \%$ apoptosis as measured by Annexin-V and PI staining). Protein lysates were subjected to immunoprecipitation with an anti-BIM antibody, and MCL-1, BCL2 and BCL-X $L_{L}$ bound to BIM were then analyzed by immunoblotting. Their levels were quantified by densitometry analysis of bands using ImageJ software, normalized to those of BIM, and depicted as bar diagrams. Whole cell lysates of each cell line are also shown. 
A

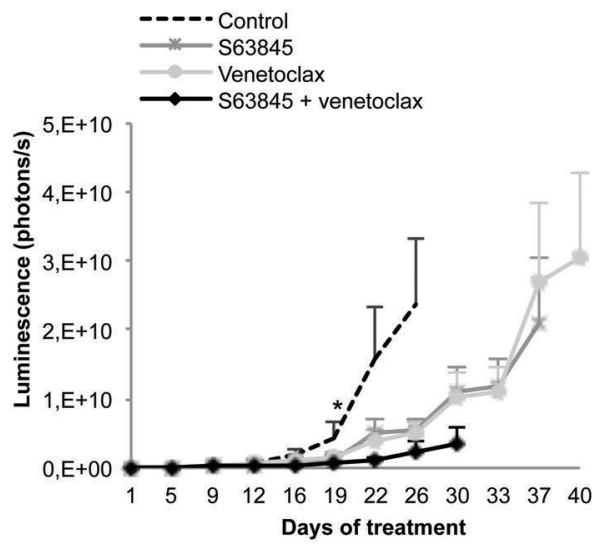

C

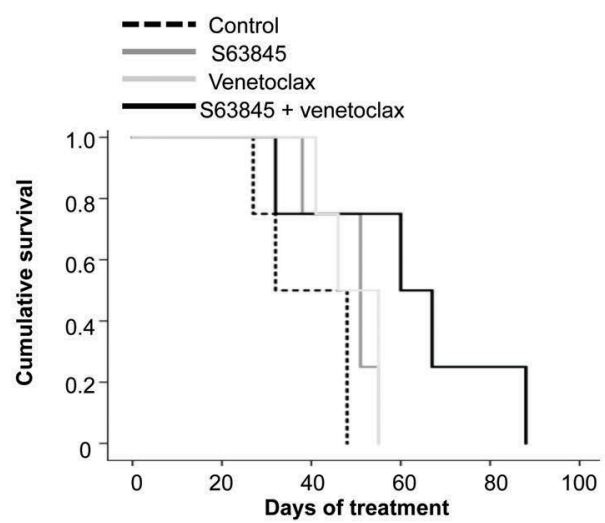

B

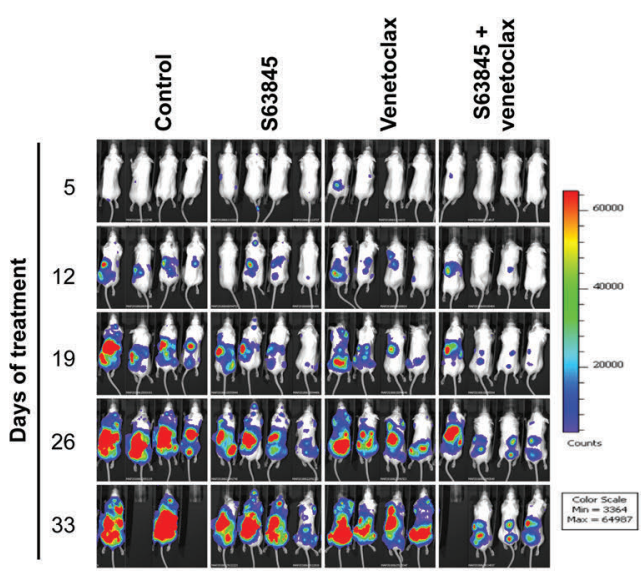

D

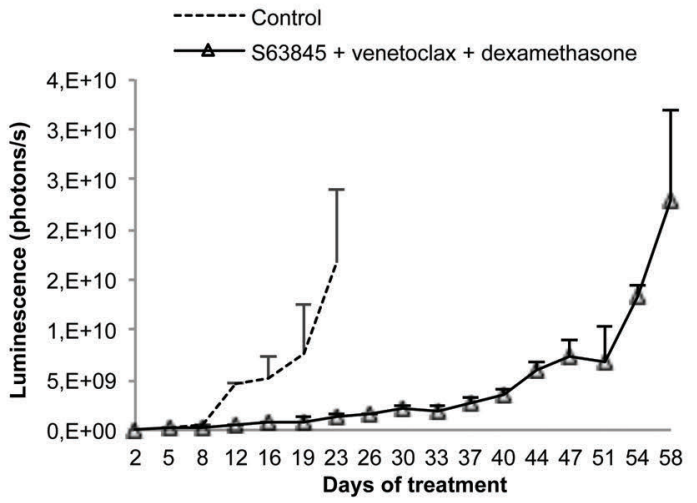

E

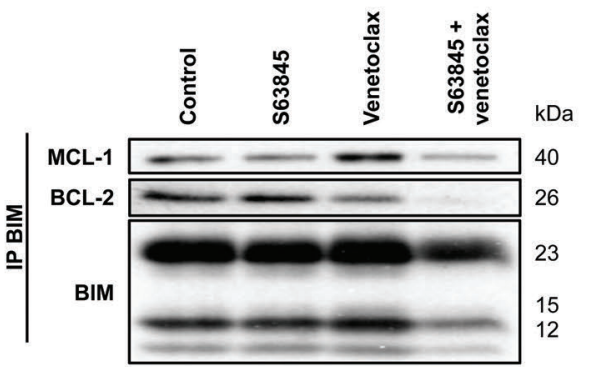

Q Control $\square \mathrm{s} 63845 \square$ Venetoclax $\square \mathrm{s} 63845$ + venetoclax

MCL-1

BCL-2
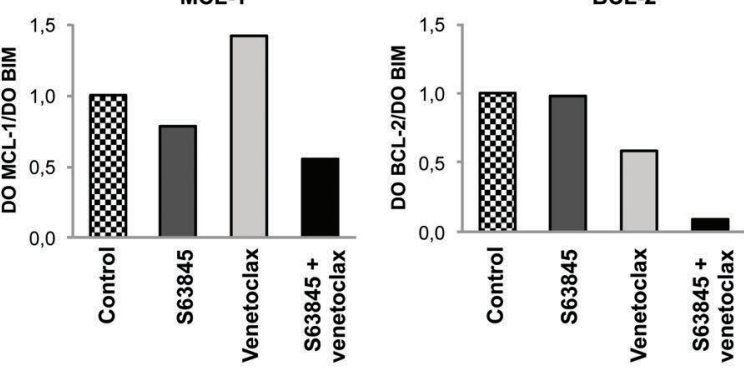

Figure 3. The S63845 + venetoclax combination has potent in vivo anti-myeloma activity. (A) In vivo efficacy of S63845+venetoclax in an RPMI-8226-luc xenograft model of disseminated multiple myeloma (MM) in BRG mice. Experimental groups included: control (vehicle), S63845 (12.5 mg/kg intravenous, week$\mathrm{ly})$, venetoclax $(100 \mathrm{mg} / \mathrm{kg}$ oral administration, 5 days per week), and the respective combination $(\mathrm{n}=4$ per group). Mice were treated until death or sacrifice for humane reasons. Statistically significant differences (Kruskal-Wallis test followed by Dunn's post-hoc comparisons, *P<0.05) were observed from day 19 onwards when comparing the combination with the control. Data are summarized as the mean \pm Standard Error of Mean (SEM). (B) Images representing the bioluminescence signal of each mouse by treatment group from day 5 to day 33 of treatment. (C) Kaplan-Meier curves representing the survival of each treatment group. (D) Efficacy of the triple combination S63845+venetoclax+dexamethasone using the doses and scheme as in (A) but with intraperitoneal dexamethasone administration ( $1 \mathrm{mg} / \mathrm{kg}, 2$ days/week) ( $\mathrm{n}=3$ per group). Data are shown as mean \pm SEM. (E) RPMI-8226 cells were subcutaneously injected in CB17-SCID mice. When plasmacytomas reached $2 \mathrm{~cm}$ in one of their diameters, animals received one dose of vehicle, S63845 (12.5 mg/kg), venetoclax (100 mg/kg) or the respective combination ( $n=2$ per group). Tumors were excised 24 hours after treatment and protein lysates from tumors were subjected to immunoprecipitation with an anti-BIM antibody. MCL-1, BCL-2 and BCL-X anti-apoptotic proteins bound to BIM were then analyzed by immunoblotting, quantified by densitometry analysis of bands normalized to those of BIM using Image J software, and represented in bar diagrams. 
been described, ${ }^{6,7} \mathrm{MM}$ cells are heavily dependent on MCL- $1^{8,9}$ and high levels of MCL-1 have been associated with venetoclax resistance. ${ }^{2,10}$ In this regard, a new selective MCL-1 inhibitor, S63845, has recently demonstrated single-agent anti-tumor effect in $\mathrm{MM} .{ }^{11}$ Within this scenario, we sought to test the potential synergistic apoptotic induction of $\mathrm{S} 63845$ and venetoclax in $\mathrm{MM}$. Mechanistically, the shift in MM-cell dependence to different anti-apoptotic proteins observed with each agent in monotherapy was greatly overcome with the double combination, translating into important anti-myeloma efficacy in vitro, ex vivo and in vivo.

We selected five myeloma cell lines with different sensitivities to S63845 and venetoclax in monotherapy (MM.1S being the most resistant and KMS12-BM the most sensitive), and evaluated the cytotoxic effect of the combination of both agents by flow cytometry (Figure 1A) and MTT assay (Online Supplementary Figure S1). Overall, our in vitro findings show that the S63845+venetoclax combination clearly increased apoptotic cell death and reduced cell viability, with combination indexes (CI) reaching a strong synergism $(0.1<\mathrm{CI}<0.3)$ in almost all cell lines (Online Supplementary Figures S2 and S3). This effect was dose- and time-dependent, and short drug exposures of 3-6 hours already triggered the apoptotic effect (Figure 1A and B). Given the clinical interest of the addition of dexamethasone in the current backbone of MM treatment, the triple combination of S63845+venetoclax+dexamethasone was also evaluated. Dexamethasone clearly increased the efficacy of both S63845 and venetoclax, and the triple combination showed an even stronger synergism than the S63845+venetoclax doublet in MM.1S (best $\mathrm{CI}=0.054$ ) and RPMI-8226 (best $\mathrm{CI}=0.099$ ) cells (Online Supplementary Figure $S 4 A$ and $B)$.

The anti-tumoral effect of $\mathrm{S} 63845$ and venetoclax was further investigated ex vivo in cells isolated from eight MM patients. Patients 1 and 2 harbored the $t(11 ; 14)$ translocation, Patients 3 to 7 had $1 \mathrm{q}$ gain, and Patient 8 did not bear any of those cytogenetic alterations. S63845 in monotherapy was active in almost all patients (Online Supplementary Figure S5A), although those patients with 1q amplification (thus harboring the locus of the MCL1 gene) were significantly more sensitive to this agent (Student $t$-test, $P<0.05)$ (Online Supplementary Figure S5B). Whether $1 \mathrm{q}$ amplification is a bona-fide marker of response to MCL-1 inhibitors is being tested in ongoing preclinical and clinical studies. On the other hand, only Patient 2 bearing the $\mathrm{t}(11 ; 14)$ translocation was clearly sensitive to venetoclax as single agent (Online Supplementary Figure S5C). This situation is consistent with the clinical data in which less than half of the $t(11 ; 14)$ patients responded to venetoclax. ${ }^{5}$ Finally, in 5 of the 8 evaluated patients (Patients 1, 2, 3, 7 and 8), the combination enhanced the apoptotic induction of both agents in monotherapy, but, interestingly, this was particularly evident in Patient 2 [venetoclax responder harboring the $\mathrm{t}(11 ; 14)$ translocation] and Patient 8 [insensitive to both drugs in monotherapy without $\mathrm{t}(11 ; 14)$ translocation or +1 q gain alterations] (Figure $1 \mathrm{C}$ ). The toxicity on normal lymphocytes was clearly lower than that on tumor cells, suggesting a therapeutic window for both drugs (Figure 1C and Online Supplementary Figure S5A-C).

Next, we explored the mechanism of action of the S63845+venetoclax combination. BIM is a pro-apoptotic protein which has already been shown to be involved in the mechanism of action of S63845 and venetoclax in monotherapy. ${ }^{12-14}$ Accordingly, using CRISPR/Cas9 gene editing in MM.1S cells, we selected BIM knock-out clones for 2 of the 3 major BIM isoforms (BIM $B I M L)$, which showed notably decreased sensitivity to the S63845+venetoclax combination (Figure 2A). Since these data prove the involvement of BIM in the mechanism of action of the double combination, the binding of MCL-1, BCL-2 and BCL-XL anti-apoptotic proteins to BIM was next explored in the MM.1S and KMS12-BM cell lines (Figure 2B and C). S63845 treatment clearly disrupted MCL-1/BIM complexes, but also induced a compensatory increase in BCL-2/BIM complexes over control levels in both cell lines. BCL-XL/BIM complexes were also increased after $\mathrm{S} 63845$ treatment in the MM.1S cell line, but these complexes were absent in KMS12-BM cells. These results imply that $S 63845$ treatment may change MM-cell dependence from MCL-1 to BCL-2, and also to $B C L-X_{L}$ in cells particularly dependent on this protein, thus suggesting a potential mechanism of resistance. On the other hand, and consistent with previous reports, ${ }^{2,13}$ venetoclax impaired the formation of BCL-2/BIM complexes and also increased the binding of MCL-1 to BIM over control levels in KMS12-BM cells and the binding of both MCL-1 and BCL-XI to BIM in MM.1S cells, suggesting a parallel situation to that observed with S63845. Importantly, after treatment with the S63845+venetoclax combination, BCL-2/BIM complexes remained low in both cell lines tested. However, in MM.1S cells, MCL-1 was still able to interact with BIM, although to a lesser extent than with venetoclax in monotherapy, thereby diminishing the previously described venetoclax escape mechanism. Regarding BCL-X工 $/$ BIM complexes, their increase with $\mathrm{S} 63845$ and venetoclax treatments in monotherapy was not further potentiated by the double combination. Whole cell lysates did not show major changes on MCL-1, BCL-2, BCL-X $\mathrm{L}$ and BIM levels in MM.1S and KMS12-BM cells treated with S63845 and venetoclax alone and in combination (Figure $2 \mathrm{~B}$ and $\mathrm{C}$ ). Finally, we immunoprecipitated MCL-1 and BCL-2 antiapoptotic proteins, and analyzed BIM binding by immunoblotting (Online Supplementary Figure S6A and B); the results obtained were in accordance with those from BIM immunoprecipitation. MCL-1/NOXA and BCL-2/PUMA complexes were also evaluated, but low expression of these pro-apoptotic proteins precluded evaluation of their role in response to the drugs (data not shown).

Furthermore, the efficacy of S63845+venetoclax was explored in vivo in an aggressive disseminated model of MM. The double treatment delayed tumor growth, and in contrast to the agents in monotherapy, produced a statistically significant benefit with respect to the control from day 19 onwards (Figure 3A). Of note, at day 32, a mouse treated with $\mathrm{S} 63845+$ venetoclax, despite only having a relatively localized bioluminescence signal, developed hind-limb paralysis and was euthanized for humane reasons (Figure 3B). Nevertheless, the efficacy in controlling tumor growth translated into improved survival of mice treated with S63845+venetoclax, with a median survival of 60 days (range: $32-88$ days) compared with 51 days for S63845 (range: 38-55 days) and 46 days for venetoclax (range: 41-55 days) (Figure 3C), although these differences were not statistically significant. Remarkably, none of the treatments caused a significant reduction in body weight (Online Supplementary Figure $S 7 A$ ) or other signs of toxicity. It should be noted that S63845 has weaker affinity for murine MCL-1, ${ }^{11}$ and therefore other models ${ }^{15}$ would be required to better evaluate the safety margins of this combination.

Similarly to in vitro studies, we also evaluated the activ- 
ity and toxicity of the triple combination of S63845+venetoclax+dexamethasone in the previously mentioned in vivo disseminated model of MM. The triple combination induced approximately 30 days delay in tumor growth compared with the control group (Figure 3D). Most importantly, the tolerability of this triple combination was excellent, without significant body weight loss (Online Supplementary Figure $S 7 B$ ) or other signs of toxicity.

Finally, we performed mechanistic studies on tumor cells from large RPMI-8226 plasmacytomas excised 24 hours after one dose of treatment (Figure 3E). In accordance with in vitro data, treatment with S63845 and venetoclax in monotherapy, respectively, impaired the binding of MCL-1 and BCL-2 to the pro-apoptotic protein BIM. Moreover, there was a compensatory upregulation of MCL-1/BIM complexes in tumors from mice treated with venetoclax, but no increase in BCL-2/BIM complexes in tumors from mice treated with S63845. Remarkably, the $\mathrm{S} 63845+v e n e t o c l a x$ combination completely disrupted BCL-2/BIM complexes and was able to counteract the compensatory upregulation of MCL-1 bound to BIM in tumors treated with venetoclax in monotherapy. Thus, in vivo, benefit is observed with the double combination relative to the disruption of BIM complexes with MCL-1 and BCL-2.

In conclusion, we have shown the high preclinical efficacy and synergism of the $\mathrm{S} 63845$ and venetoclax combination on MM cells, mediated at least in part by the simultaneous inhibition of the binding of MCL-1 and BCL-2 to BIM. Our preclinical results provide a strong rationale for the clinical investigation of the combination of an MCL-1 inhibitor with venetoclax for the treatment of $\mathrm{MM}$ patients. In addition, based on the preliminary results obtained with the triple combination, the addition of dexamethasone may also be considered.

Esperanza M Algarin, Andrea Díaz-Tejedor,

Pedro Mogollón, Susana Hernández-Garcia,"

Luis A. Corchete, Laura San-Segundo, ${ }^{1}$

Montserrat Martín-Sánchez, Lorena González-Méndez, ${ }^{1}$

Marie Schoumacher, ${ }^{2}$ Sebastien Banquet, ${ }^{2}$

Laurence Kraus-Berthier, ${ }^{2}$ Ioana Kloos, ${ }^{2}$ Alix Derreal, ${ }^{2}$

Ensar Halilovic, ${ }^{3}$ Heiko Maacke, ${ }^{4}$ Norma C. Gutiérrez,

María-Victoria Mateos, ${ }_{1}$ Teresa Paino, ${ }^{1}$ Mercedes Garayoa ${ }^{4}$

and Enrique M. Ocio ${ }^{1,5^{*}}$

*MG and EMO contributed equally to this work.

University Hospital of Salamanca (IBSAL)-Cancer Research Center (IBMCC-CSIC-USAL), Salamanca, Spain; Institut de Recherches Servier, Suresnes, France; ${ }^{3}$ Novartis Institutes for Biomedical Research, Cambridge, MA, USA; ${ }^{4}$ Novartis Institutes for Biomedical Research, Basel, Switzerland and 'University Hospital Marqués de Valdecilla (IDIVAL); University of Cantabria, Santander, Spain

Correspondence: ENRIQUE M. OCIO

ocioem@unican.es

doi:10.3324/haematol.2018.212308
Funding: this work was supported by the Spanish ISCIII-FIS and FEDER Funds (PI 15/00067 and PI 15/02156) and the Regional Health Council of Castilla y Léon (GRS 1604/A/17). EMA was supported by a grant from the Regional Education Council of Castilla y Léon co-financed by the European Social Fund.

Information on authorship, contributions, and financial \& other disclosures was provided by the authors and is available with the online version of this article at www. haematologica.org.

\section{References}

1. Witzig T, Timm M, Larson D, Therneau T, Greipp P. Measurement of apoptosis and proliferation of bone marrow plasma cells in patients with plasma cell proliferative disorders. Br J Haematol. 1999;104(1):131-137.

2. Touzeau C, Dousset C, Le Gouill S, et al. The Bcl-2 specific BH3 mimetic ABT-199: a promising targeted therapy for $\mathrm{t}(11 ; 14)$ multiple myeloma. Leukemia. 2014;28(1):210-212.

3. Vogler M, Walter HS, Dyer MJS. Targeting anti-apoptotic BCL2 family proteins in haematological malignancies - from pathogenesis to treatment. Br J Haematol. 2017;178(3):364-379.

4. Souers AJ, Leverson JD, Boghaert ER, et al. ABT-199, a potent and selective BCL-2 inhibitor, achieves antitumor activity while sparing platelets. Nat Med. 2013;19(2):202-208.

5. Kumar S, Kaufman JL, Gasparetto C, et al. Efficacy of venetoclax as targeted therapy for relapsed/refractory $\mathrm{t}(11 ; 14)$ multiple myeloma. Blood. 2017;130(22):2401-2409.

6. Gomez-Bougie P, Maiga S, Tessoulin B, et al. BH3-mimetic toolkit guides the respective use of BCL2 and MCL1 BH3-mimetics in myeloma treatment. Blood. 2018;132(25):2656-2669.

7. Touzeau C, Ryan J, Guerriero J, et al. BH3 profiling identifies heterogeneous dependency on Bcl-2 family members in multiple myeloma and predicts sensitivity to BH3 mimetics. Leukemia. 2016;30(3):761764

8. Gong J-N, Khong T, Segal D, et al. Hierarchy for targeting prosurvival BCL2 family proteins in multiple myeloma: pivotal role of MCL1. Blood. 2016;128(14):1834-1844.

9. Derenne S. Antisense strategy shows that $\mathrm{Mcl}-1$ rather than $\mathrm{Bcl}-2$ or $\mathrm{Bcl}-\mathrm{xL}$ is an essential survival protein of human myeloma cells. Blood. 2002;100(1):194-199.

10. Dousset C, Maïga S, Gomez-Bougie P, et al. BH3 profiling as a tool to identify acquired resistance to venetoclax in multiple myeloma. $\mathrm{Br}$ J Haematol. 2017;179(4):684-688.

11. Kotschy A, Szlavik Z, Murray J, et al. The MCL1 inhibitor S63845 is tolerable and effective in diverse cancer models. Nature. 2016;538(7626):477-482.

12. Morales AA, Kurtoglu M, Matulis SM, et al. Distribution of Bim determines Mcl-1 dependence or codependence with Bcl-xL/Bcl-2 in Mcl-1-expressing myeloma cells. Blood. 2011;118(5):1329-1339.

13. Punnoose EA, Leverson JD, Peale F, et al. Expression Profile of BCL2, BCL-XL, and MCL-1 Predicts Pharmacological Response to the BCL-2 Selective Antagonist Venetoclax in Multiple Myeloma Models. Mol Cancer Ther. 2016;15(5):1132-1144.

14. Merino D, Whittle JR, Vaillant F, et al. Synergistic action of the MCL1 inhibitor S63845 with current therapies in preclinical models of triple-negative and HER2-amplified breast cancer. Sci Transl Med. 2017;9(401):eaam7049.

15. Brennan MS, Chang C, Dewson G, et al. Humanized Mcl-1 mice enable accurate pre-clinical evaluation of MCL-1 inhibitors destined for clinical use. Blood. 2018;132(15):1573-1583. 